\title{
Comparison of Various Security Features of Genuine, Scanned and Photocopied Indian Currency Note of the Denomination 2000
}

\author{
Anjali $\mathrm{A}^{1}$, Abhishek $\mathrm{A}^{1}$, Uttam $\mathrm{S}^{2}$ and Priyanka $\mathrm{V}^{*_{2}}$ \\ ${ }^{1}$ MSc. Forensic Science, Chandigarh University, Gharuan, Mohali, India \\ ${ }^{2}$ Assistant Professor, Chandigarh University, Gharuan, India
}

${ }^{*}$ Corresponding author: Priyanka V, Assistant Professor, Chandigarh University, Gharuan, India, E-mail: priyankakverma25@gmail.com

Citation: Anjali A, Abhishek A, Uttam S, Priyanka V (2017) Comparison of Various Security Features of Genuine, Scanned and Photocopied Indian Currency Note of the Denomination 2000. J Forensic Sci Criminol 5(3): 305

Received Date: March 23, 2017 Accepted Date: August 22, 2017 Published Date: August 23, 2017

\begin{abstract}
A banknote is a type of a negotiable instrument known as a promissory note, made by a bank, payable to the bearer on demand. The Indian Rupee is the official currency of the Republic of India. The issuance of the currency is controlled by the Reserve Bank of India. In this modern epoch, circulation of counterfeit currency has greatly augmented as the most common unlawful activity. To stop this illegal transmission, general public must accustomed themselves with genuinely of the currency notes of the denomination value 2000.

This paper made an effort to aid people to differentiate between the fake currency and the genuine currency by excavating various security features of the highest denomination and comparing the same with the scanned and photocopied version of the denomination. Features such as Micro-printing, Optically Variable ink, optic fibres, Intaglio printing, latent image and in numerous magic image (spiral pattern, nuclear pattern, bird, etc.) remained intact when the denomination is photocopied and scanned.

Forgery of currency of denomination 2000 will be a challenging task for the perpetrators as many features are embedded in the paper which makes the currency of highest denomination more secure and non-vulnerable.
\end{abstract}

Keywords: Currency; Security Features; Indian Bank Note; 2000; RBI; fake; Genuine

\section{Introduction}

The main objective of the present study is to spread cognizance amongst the general public about the SEVERAL SIGNIFICANT SECURITY features of the highest denominational currency note introduced recently. This would help the people to differentiate between the genuine note and the fake ones. Also, it tends to showcase the hidden features of the note that would rather make the counterfeiting of this note difficult and nearly impossible. A detailed comparison of the security features present in the genuine, scanned and photocopied currency note is very well explained in this paper.

Aimed at combating corruption and black money, the arrival of the new high-value currency note has come as a surprise for everybody. The size of the new ₹ 2000 note is $66 \mathrm{~mm} \mathrm{X} 166 \mathrm{~mm}$ and is available in a magenta base colour (Figure 1A and 1B). It bears a portrait of Mahatma Gandhi in the front and a motif of the Mangalyaan on reverse side of the note illustrating the country's first project in interplanetary space [1]. RBI has issued two sets of notes, one with the inset letter ' $R$ ' and one without the inset letter [2].

\section{Need of Issuing the Indian Currency Note of Denomination Value 2000}

The new currency note of this high denominational value was dispatched by the RESERVE BANK OF INDIA with the main purpose of curbing black money. Its release has been announced in such a way that the corrupting elements are unable to prepare themselves in any way to counter this sudden 'financial catastrophe. The levels of corruption have reached such a stage that it is estimated that the black money accumulated in India, in various shapes and forms, is worth around billions of dollars. In this way, the Indian Government is endeavouring to bring all this unaccounted currency back into the system [3].

\section{Material and Methodology}

The Indian currency note of denominational value 2000 was analysed for various features including the security features by using a hand lens/magnifier and a UV trans-illuminator. The original currency was compared with the features observed in coloured 
photocopy of the original currency note and colour printout of the scanned currency note. The note was held at different angles to observe and record the features that became visible only when tilted. The fluorescent features were analysed by studying the currency note under a UV trans-illuminator (Figure 1C and 1D). The features observed in the genuine, photocopied and scanned currency note were recorded in the observation table. The present study was conducted in the month of November when the temperature varied from $15{ }^{\circ} \mathrm{C}$ to $25^{\circ} \mathrm{C}$ and the relative humidity was between 25 to $35 \%$.

\section{Observations}

Glimpse of both the sides of the denomination 2000 under day light and UV- Trans Illuminator.

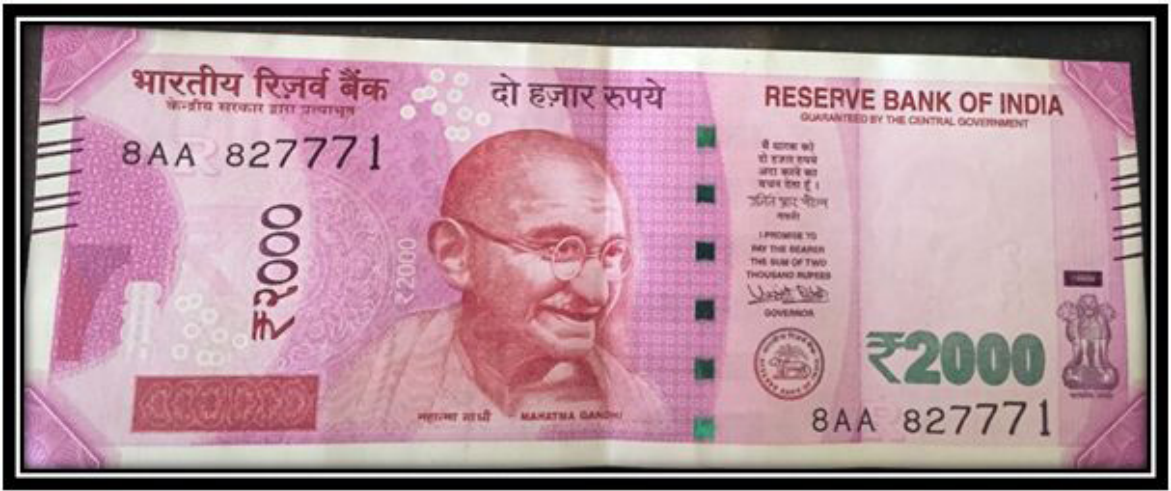

Figure 1A: Obverse side of the note under day light

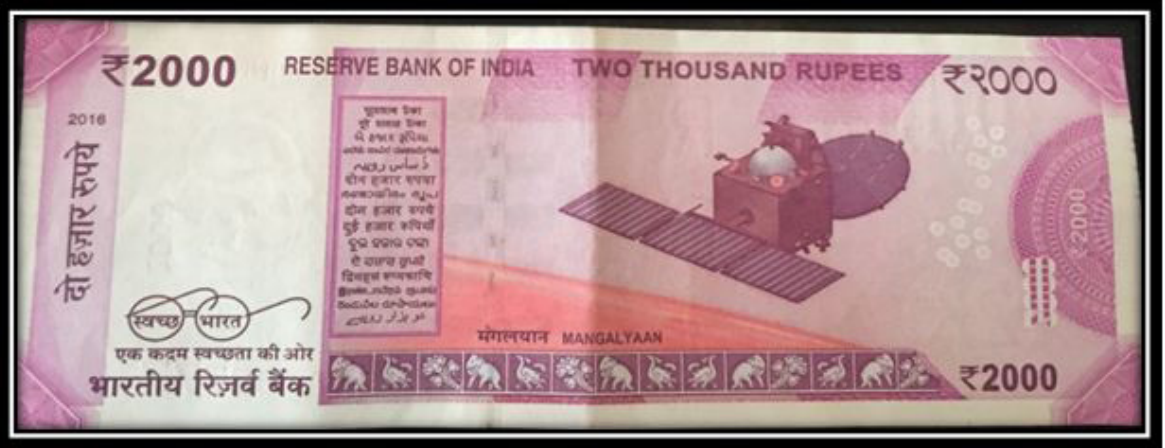

Figure 1B: Reverse side of the note under day light

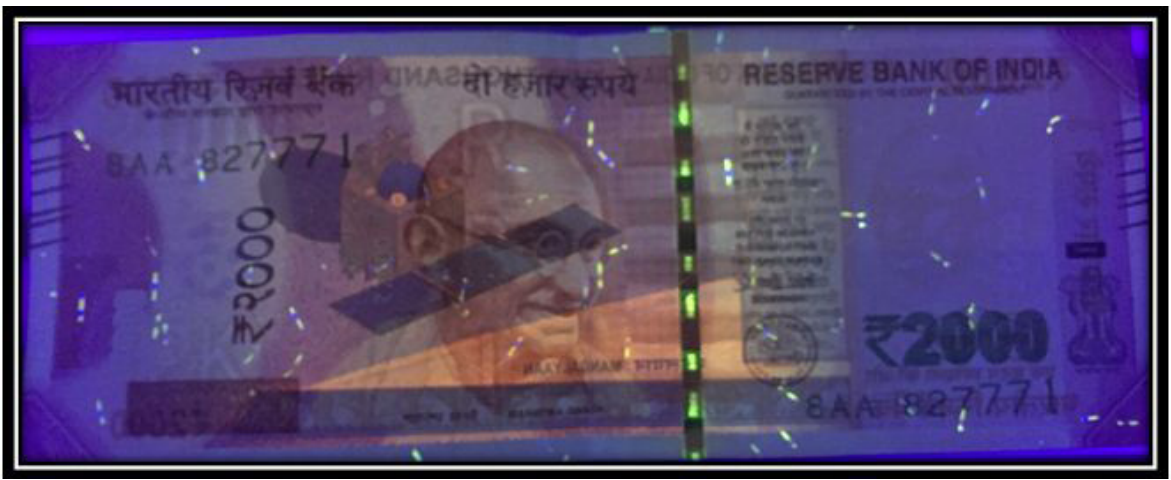

Figure 1C: Obverse side of the note under UV trans-illuminator

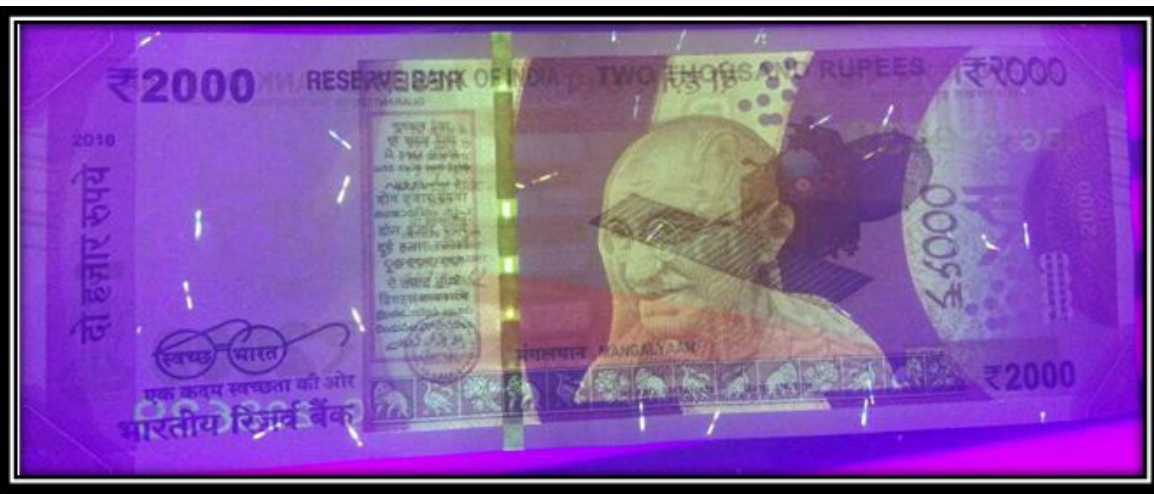

Figure 1D: Reverse side of the note under UV trans-illuminator 


\section{Security Features in the Genuine Currency Note of Denomination Value 2000}

Security features are usually incorporated in a variety of subjects and papers like the currency notes, passports, educational certificates, receipts, stamps, government documents and many more things [4]. Security features are the various authenticating features that have been incorporated in the Indian currency notes of various denomination value in order to protect them for counterfeiting. Although it is very difficult to copy and forge these security features with precision, various attempts have still been made by the forgers to counterfeit the currency notes to the possible limits. In order to curb these illegal activities of the counterfeiters and to avoid such forgeries and being cheated, it is very important for the people to have an idea about the various security features that are present in the Indian currency notes and the various other documents containing these features. The various security features present in the Indian currency note of denomination value ₹ 2000 and those observed in the photocopied and scanned currency notes are discussed in this present study.

\section{Security Features Section}

Watermarks: watermarks are the designs that are generated in the currency notes incorporated during the manufacturing process by varying the distribution of fibres in the given space. The various watermarks that are present in the 2000 denomination note are [4]

(a) Mahatma Gandhi Portrait (Figure 2A)

(b) $2 \mathrm{~K}$ Watermark (Figure 2B)

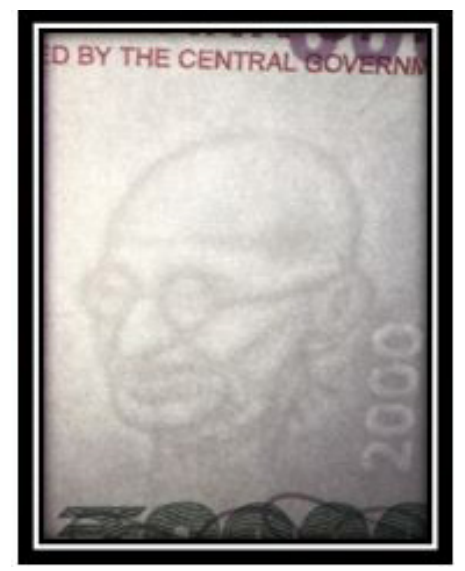

Figure 2A: Mahatma Gandhi watermark

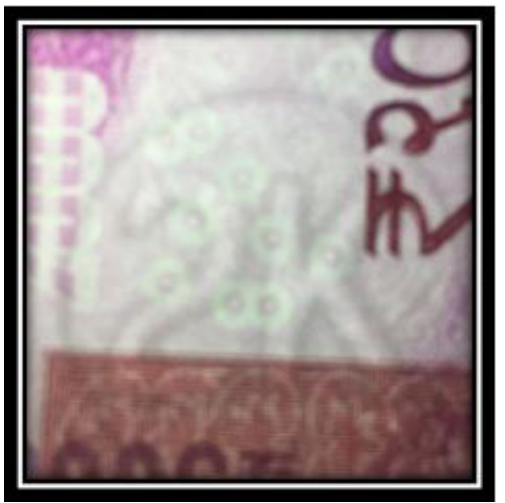

Figure 2B: 2K watermark

(c) RBI Watermark (Figure 2C)

(d) 2000 WATERMARK (Figure 2D)

(e) Electrotype Watermark (Figure 2E)

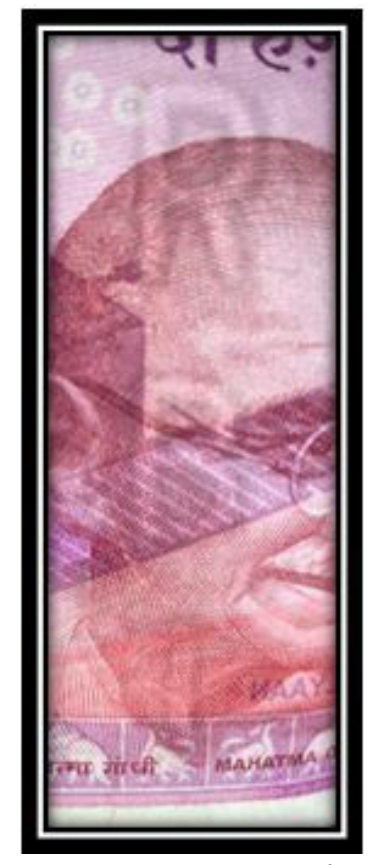

Figure 2C: RBI watermark

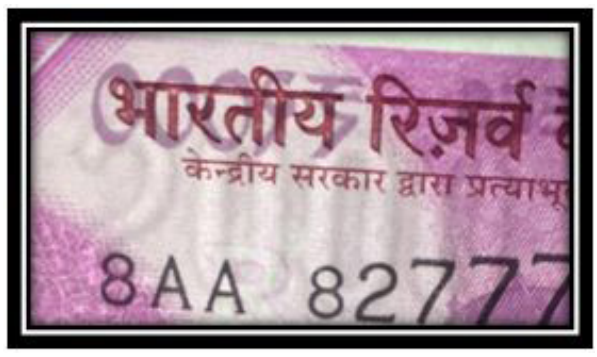

Figure 2D: 2000 watermark

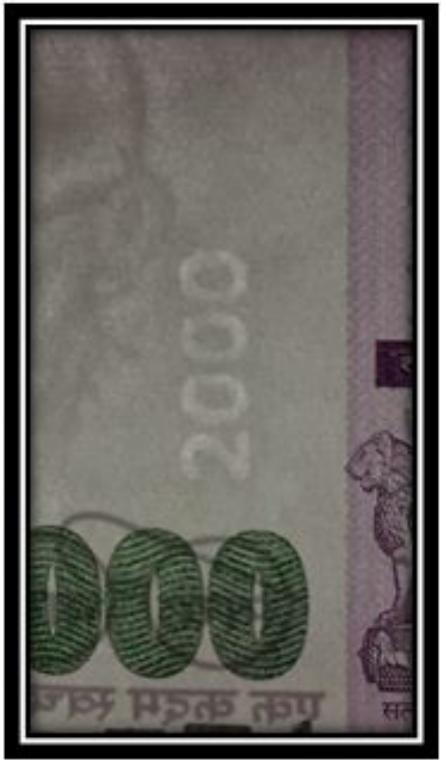

Figure 2E: Electrotype watermark 
See through Register: The see through register refers to the denomination numeral 2000 that is printed on both sides of the note, on one side, it is hollow and on the other side it is filled up. It is written horizontally just along the micro lettering and above the latent image on the left side of the currency note. It looks like one single design when seen against the light [5] (Figure 3A, 3B and 3C).

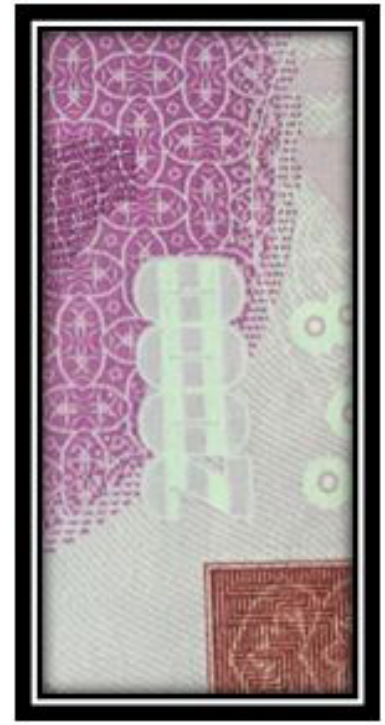

Figure 3A: See through register obverse side

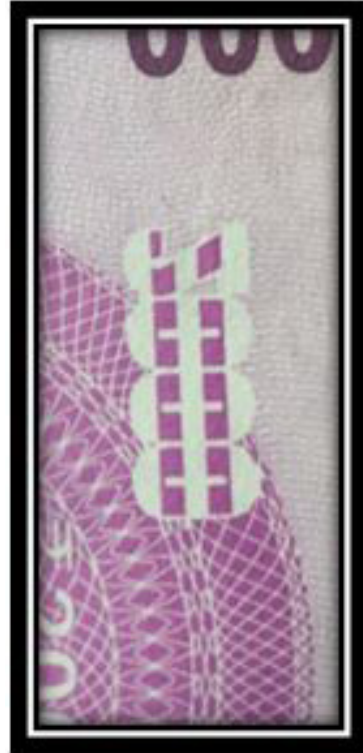

Figure 3B: See through register reverse side

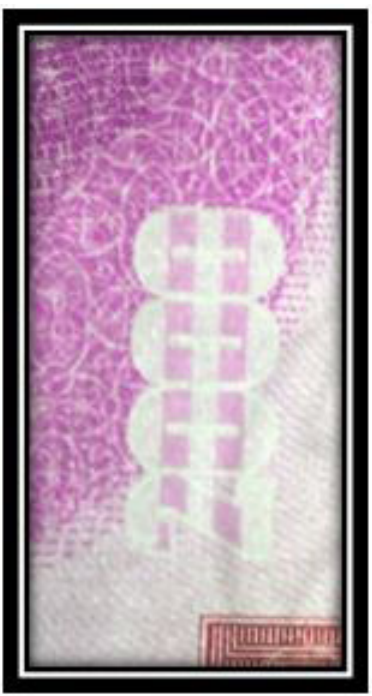

Figure 3C: See through register both obverse \& reverse

Bleed Lines: Seven angular bleed lines in 5 sets of 1-2-1-2-1 are printed on the obverse in both, the upper left and the right hand edge of the notes to aid the visually impaired (Figure 4).

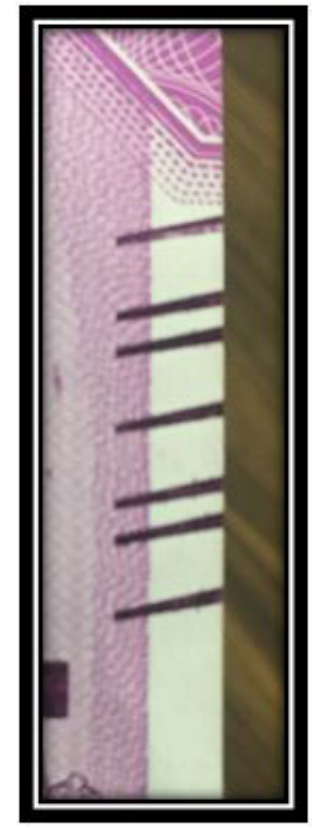

Figure 4: Bleed lines

Identification Mark: Identification marks are made on banknotes to help the visually impaired identify the denomination of notes. On Indian banknote of denomination value 2000 it appears on the right side of the watermark window. The mark in this denomination is a rectangle located above the Ashoka Pillar Emblem on the right side and has 2000 numeral written in the rectangle [5] (Figure 5).

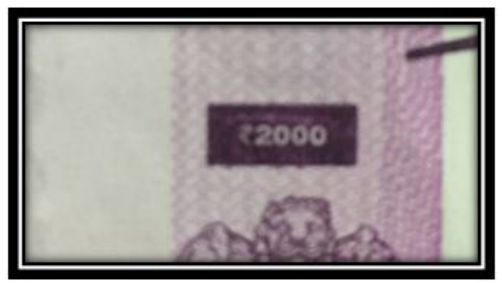

Figure 5: Identification mark 
Micro lettering: micro lettering refers to the minute inscriptions that can only be read under a microscope or by using a hand lens/ magnifier. On the Indian currency note of denomination value 2000 are encrypted "RBI 2000 INDIA". These words are written alternatively on the left side of the currency note [5] (Figure 6).

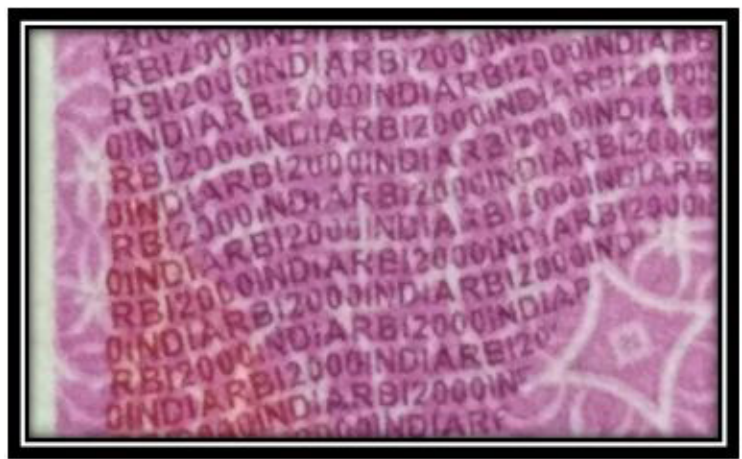

Figure 6: Micro lettering

Omron Feature: Omron feature refers to the circle-shaped anti-copy feature located on the left side of the note just above the latent image (Figure 7).

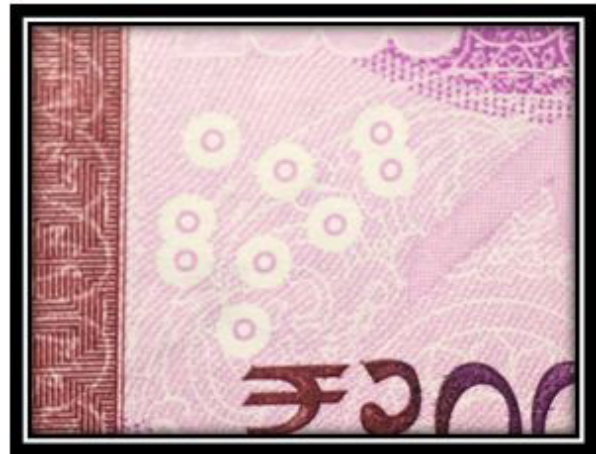

Figure 7: Omron feature

Optically Variable Ink: The denomination value 2000 in the Mahatma Gandhi watermark window of the 2000 currency note is printed on the obverse using optically variable ink. The numerals change its colour when viewed at various angles (Figure 8A and 8B).

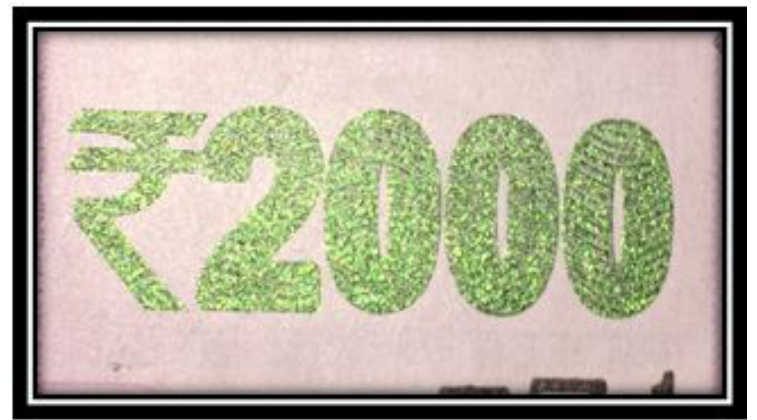

Figure 8A: Optically variable ink (green)

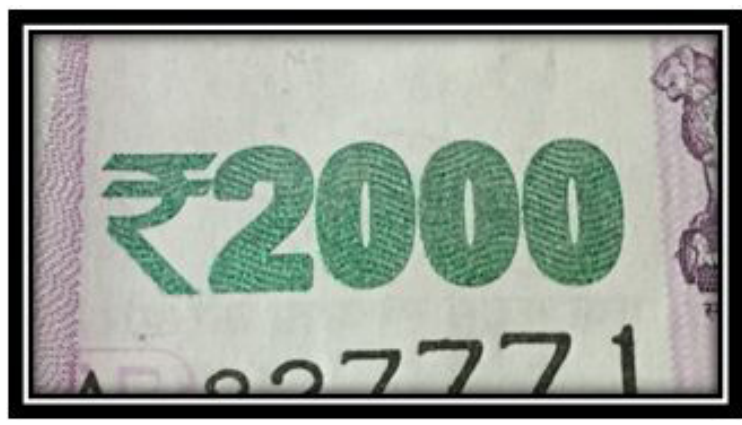

Figure 8B: Optically variable ink (blue)

Intaglio Printing: Inscriptions or motifs printed using the Intaglio printing or raised printing technique can be felt by touch. On 
Indian banknote of denomination ₹ 2000, the portrait of Mahatma Gandhi, the Reserve Bank seal, guarantee and promise clause, Ashoka Pillar Emblem on the right, RBI Governor's signature are printed in intaglio (Figure 9A, 9B, 9C, 9D and 9E).

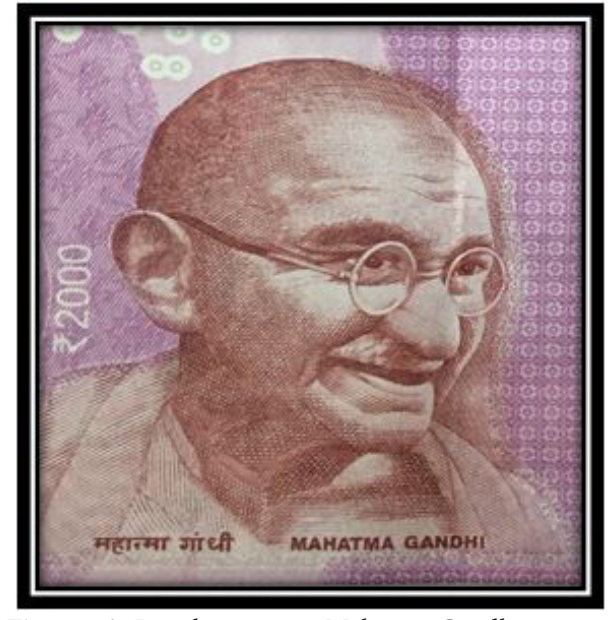

Figure 9A: Intaglio printing Mahatma Gandhi portrait

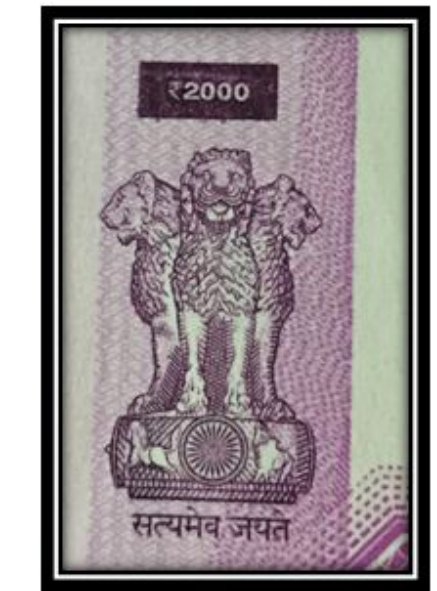

Figure 9B: Intaglio printing Ashoka emblem

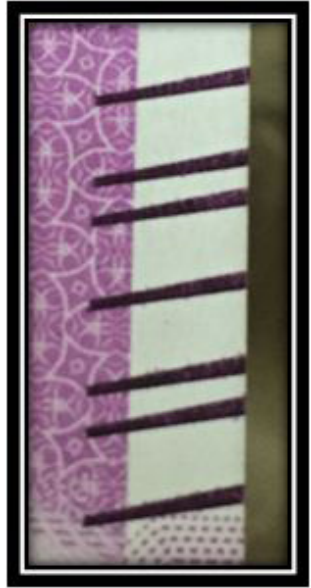

Figure 9C: Intaglio printing bleed lines

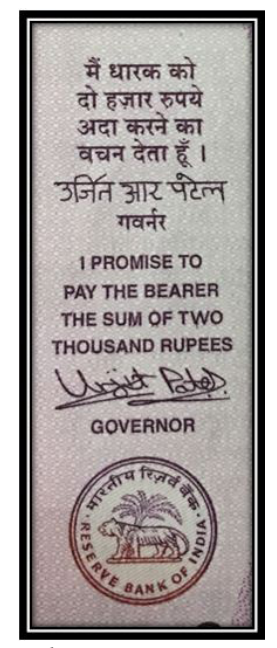

Figure 9D: Intaglio printing guarantee and clause, reserve bank seal, governor's sign

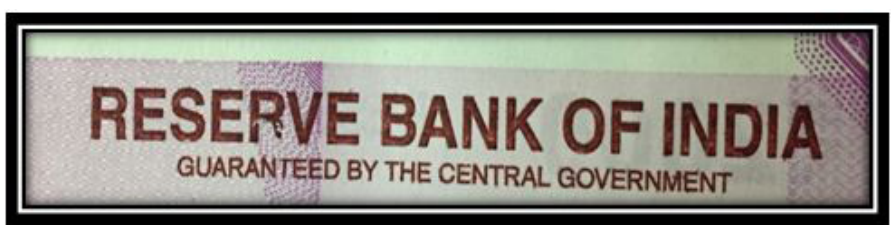

Figure 9E: Intaglio printing reserve bank of India

Security Thread: The security thread in the 2000 denomination currency note is a $3 \mathrm{~mm}$ wide strip, usually 6 windowed and reads three words particularly "RBI", "BHARAT" and "2000" imprinted on it alternatively with the security thread changing its colour from green to blue on being held at an angle and tilted [4] (Figure 10A, 10B, 10C and 10D).
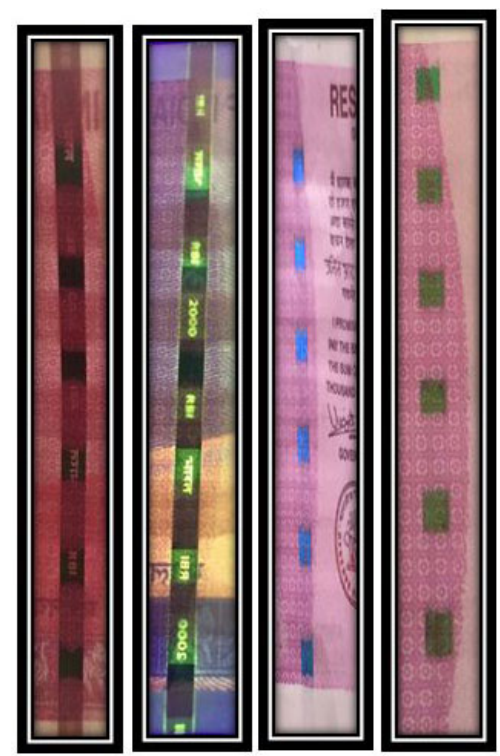

Figure 10A,B,C and D: Security thread when viwed at different light/ angles 
Optic Fibres: Optic fibres are the small fibres that are randomly distributed on both the obverse and reverse of the currency note. These fibres, only visible when the note is viewed under a UV trans-illuminator, are yellow, blue and orange in colour (Figure 11).

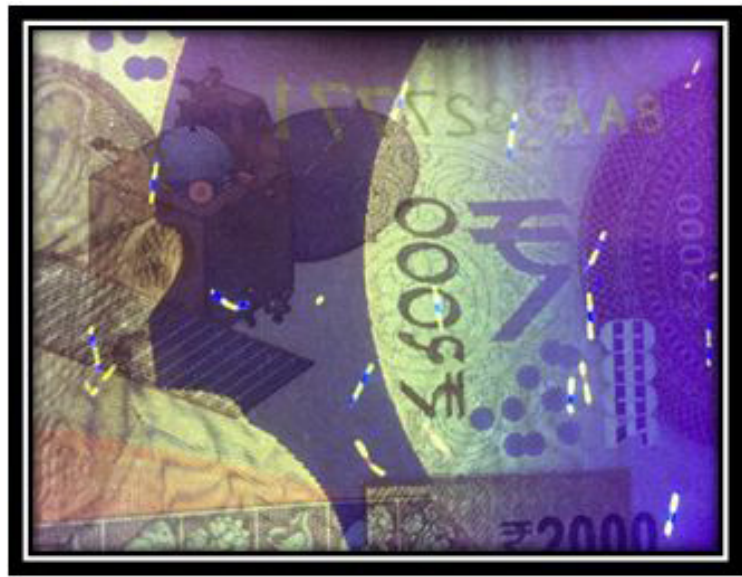

Figure 11: Optical fibres (yellow, blue, red)

New Numbering Pattern: The numbers in both the number panels tends to increase from left to right while the first three alpha numeric characters remains constant in size (Figure 12A and 12B).

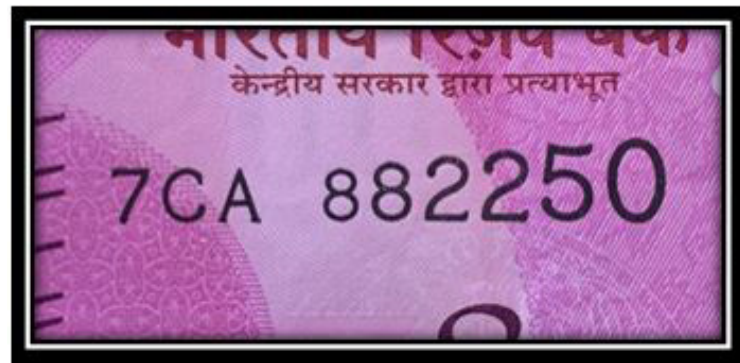

Figure 12A: Number panel without inset letter

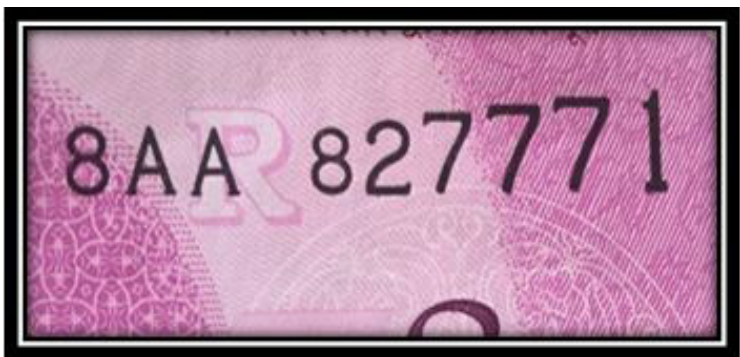

Figure 12B: Number panel with inset letter $R$

Inset Letter: The inset is a capital letter and appears on the number panel and is indicative of the printing press where the notes have been printed. Presence or absence of the same does not guarantee the authentication of the denomination (Figure 12A and 12B).

Latent Image: The latent image is a safety feature that is covered within the note. It is visible only when it is held horizontally at eye level. The obverse of Rs.2000 currency note come with a horizontal band towards the down left of the currency note and encompasses a latent image featuring the denominational value in numeral (Figure 13).

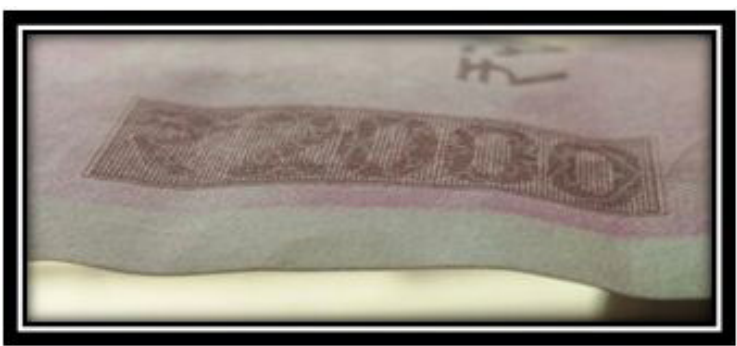

Figure 13: Latent image with 2000 denomination

Dual Usage of Ink: The Devanagari written denominational value 2000, the micro lettering portion on the left and the RBI seal have been printed in variable ink showing red and violet colouration (Figure 14A and 14B).

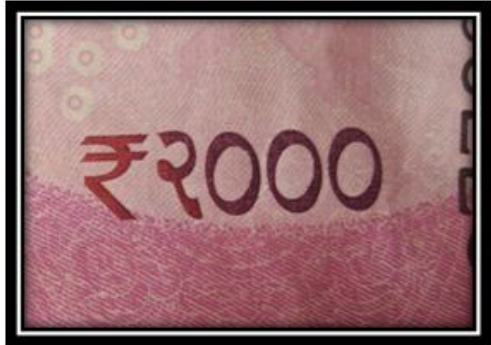

Figure 14A: Dual usage ink in Devanagari written denomination value 2000

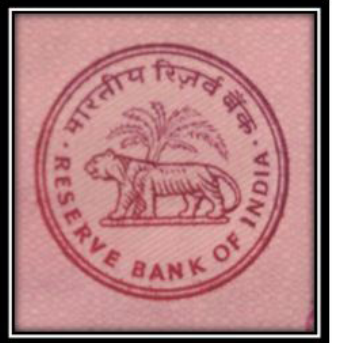

Figure 14B: Dual usage ink in the RBI seal 
Language Panel: On the reverse side of the note is present the language panel displaying the denomination of the note in 15 of the 22 official languages of India. The languages are displayed in alphabetical order- Assamese, Bengali, Gujarati, Kannada, Kashmiri, Konkani, Malayalam, Marathi, Nepali, Oriya, Punjabi, Sanskrit, Tamil, Telugu and Urdu (Figure 15).

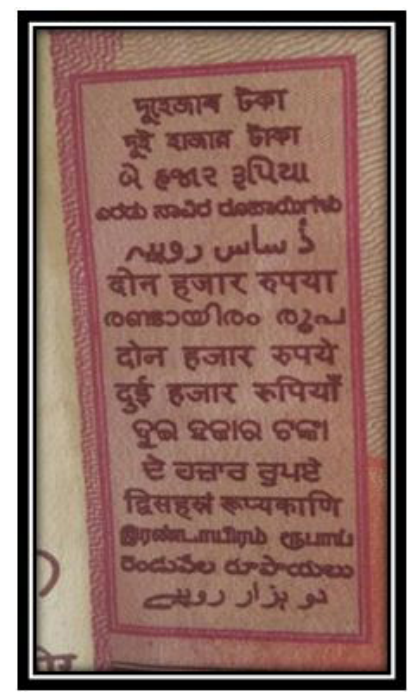

Figure 15: Language panel

Motifs: Motifs of elephant, peacock and lotus are printed on the bottom of the reverse side of the note (Figure 16).

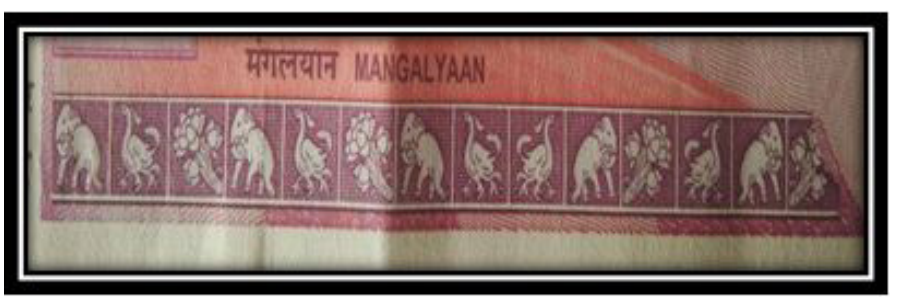

Figure 16: Motif of elephant, peacock and lotus

Geometrical Patterns: The note exhibits other designs and geometrical patterns aligning with the overall colour scheme. The geometric pattern on the left and the right corner of the note when joined forms a complete pattern (Figure 17).

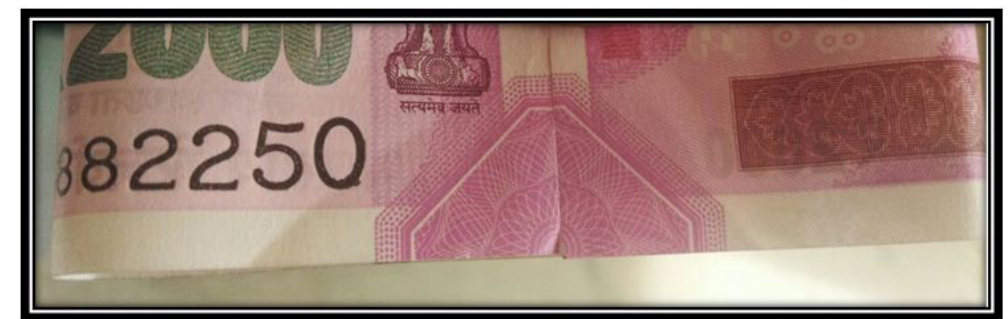

Figure 17: Geometrical patterns on all four corners of the obverse and the reverse of the note

2000 Denomination Numeral: The denominational numeral 2000 is written 6 times above and on the right side of the language panel (Figure 18).

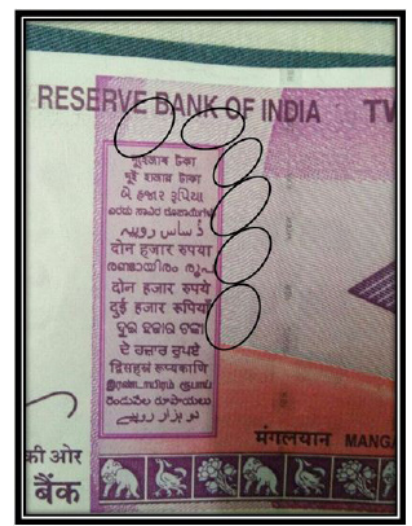

Figure 18: Denomination numeral 2000 written 6 times above and on the right side of the language panel 
The words INDIA BHARAT (Hindi) are alternatively inscribed on the left and right side of the clothes of Mahatma Gandhi near the neck (Figure 19A and 19B).
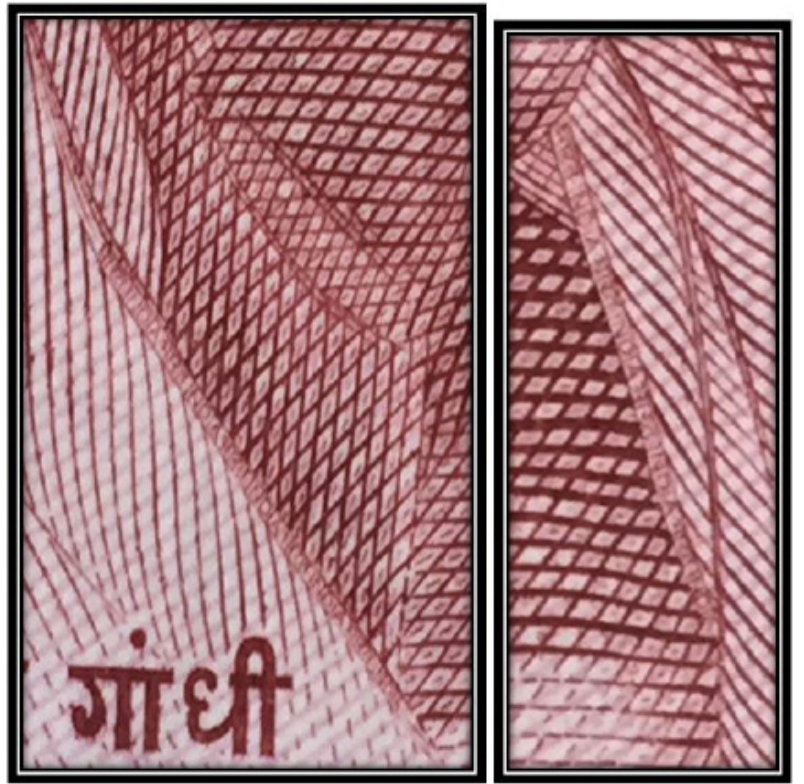

Figure 19A and B: The words India Bharat (in Hindi) written on the left and right side of the clothes of Mahatma Gandhi towards the neck

The word RBI is written on the inside of the frame of the spectacles of Mahatma Gandhi (Figure 20).

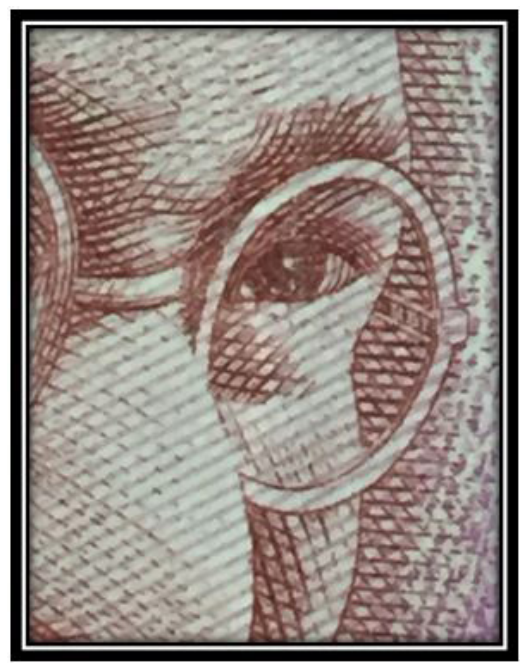

Figure 20: RBI printed on the frame of the spectacles of Mahatma Gandh

Magic Images behind Motif of Mangalyaan: Full view of the background of the motif of the Mangalyaan is shown in (Figure 21A).

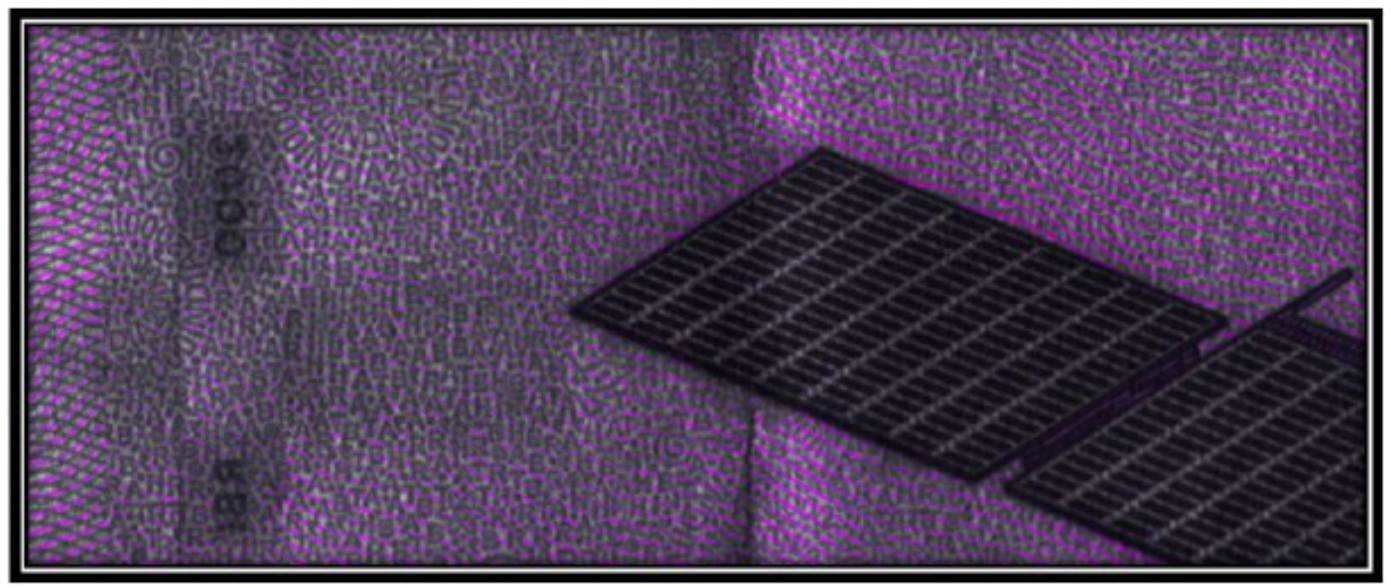

Figure 21A: Full view of the background of the motif mangalyaan 
The words INDIA RBI 2000, BHARAT are written in a circular pattern in the background of the Mangalyaan motif on the reverse side of the currency note (Figure 21B).

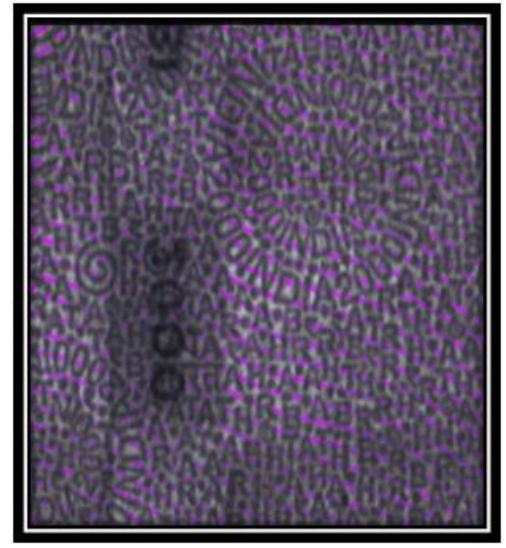

Figure 21B: The words India 2000, RBI, Bharat written in circular pattern

Various patterns like presence of bird, spiral pattern, nuclear pattern is observed on the reverse side of the note and is printed all over the area when viewed with different camera filters (Figure 21C,D and 21E).
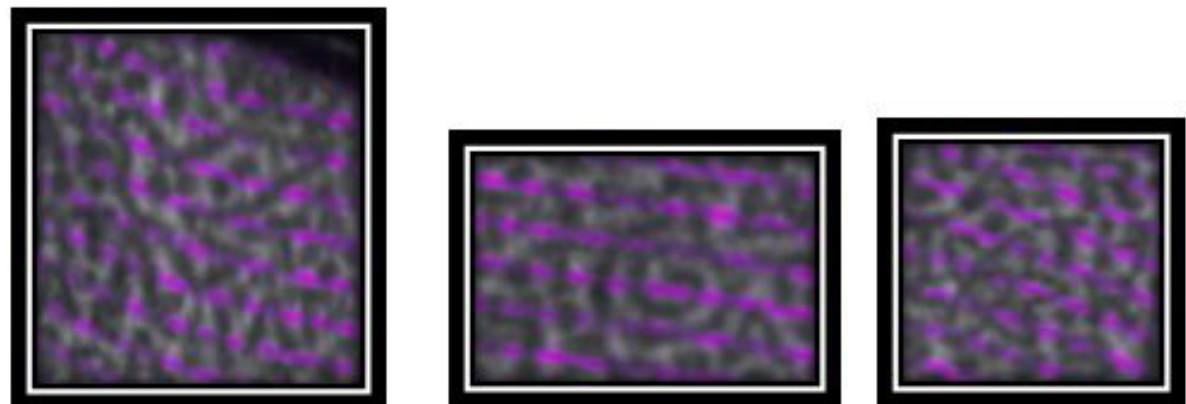

Figure 21C,D and E: Represents bird, spiral pattern, nuclear pattern respectively

\section{Photocopied and Scanned Version of 2000 Currency Note}

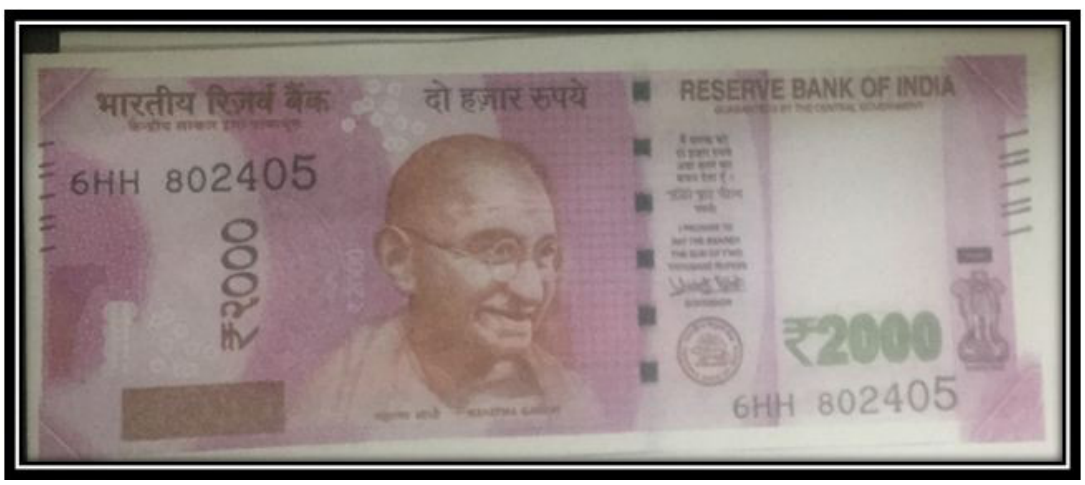

Figure 22A: Obverse side of the scanned copy of the currency note

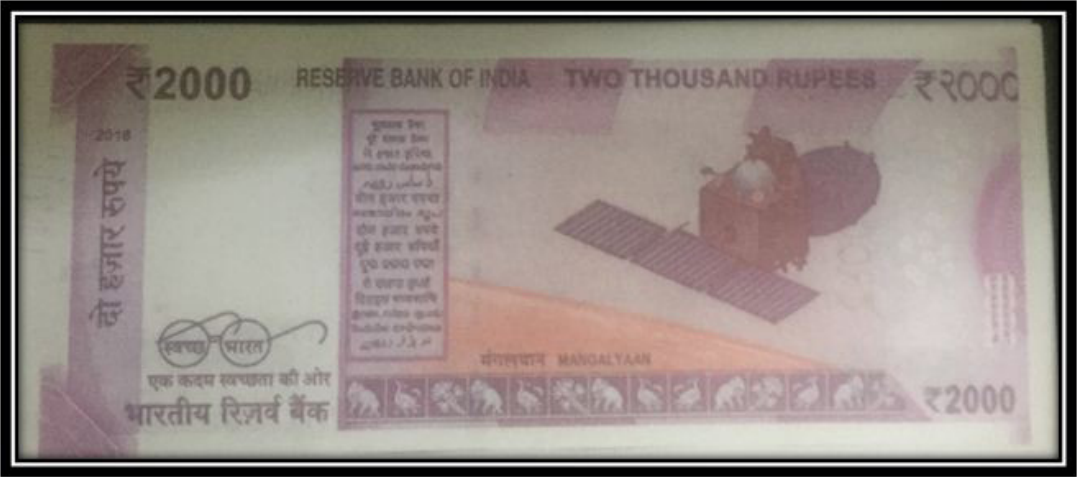

Figure 22B: Reverse side of the scanned copy of the currency note 


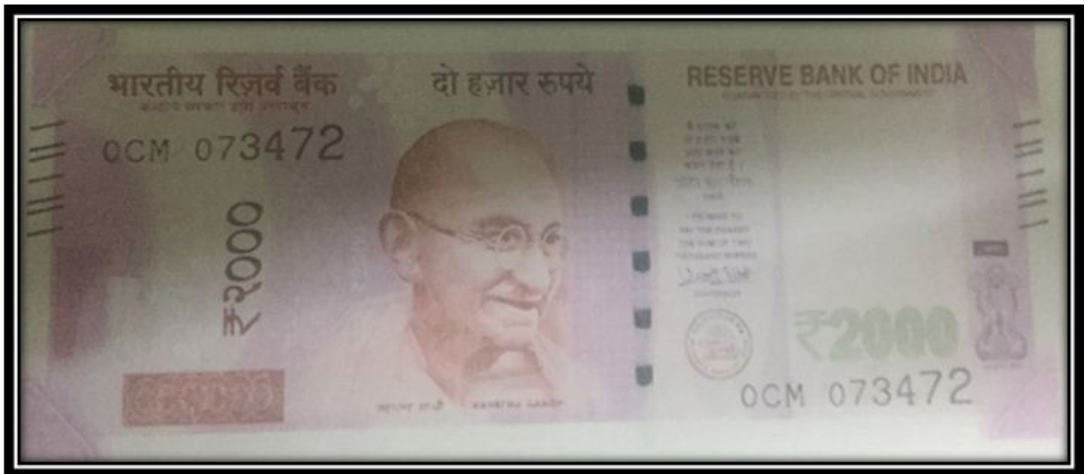

Figure 23A: Obverse side of photocopied currency note

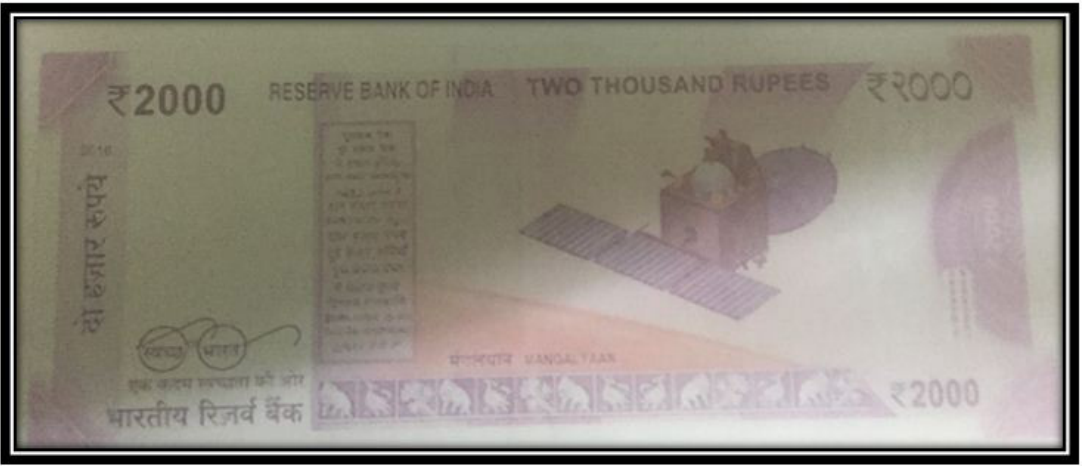

Figure 23B: Reverse side of the photocopied currency note

\section{Comparison of Features Observed in Genuine, Photocopied and Scanned Currency Notes of Denomination Value 2000}

\begin{tabular}{|c|c|c|}
\hline Features that Survived & Features that Partially Survived & Features that Do not Survived \\
\hline $\begin{array}{l}\text { Mahatma Gandhi portrait } \\
\text { watermark }\end{array}$ & RBI watermark & $\begin{array}{c}\text { Variable ink (of } 2000 \text { written in } \\
\text { Devnagri style) }\end{array}$ \\
\hline $2 \mathrm{~K}$ watermark & & $\begin{array}{l}\text { Blind Identification mark (not } \\
\text { raised) }\end{array}$ \\
\hline 2000 watermark & & Omron feature \\
\hline Electrotype watermark & & $\begin{array}{l}\text { Security thread (colour does } \\
\text { not changed, when viewed at } \\
\text { different angles) }\end{array}$ \\
\hline Micro lettering & & New numbering pattern \\
\hline $\begin{array}{l}\text { Optically variable ink (both for } \\
2000 \text { and security thread) }\end{array}$ & & Inset letter \\
\hline $\begin{array}{l}\text { The word RBI BHARAT (in } \\
\text { Hindi) on security thread }\end{array}$ & & See through register \\
\hline Latent image & & Bleed lines (not raised) \\
\hline Intaglio printing & & Language panel \\
\hline Optic fibres & & Motifs \\
\hline 2000 denomination numeral & & $\begin{array}{l}\text { Geometrical pattern (clarity is } \\
\text { reduced) }\end{array}$ \\
\hline \multicolumn{3}{|l|}{$\begin{array}{l}\text { The word INDIA BHARAT on } \\
\text { clothes of Mahatma Gandhi }\end{array}$} \\
\hline $\begin{array}{l}\text { Background of Mangalyaan } \\
\text { motif }\end{array}$ & & \\
\hline
\end{tabular}

\section{Summary}

The present study focuses on the as many as twenty security features that makes the currency note of highest denomination more safe guard and secure. It outlines and has incorporated many new traits like- all new magenta colour of the note, first time usage of dual shift ink colour, Devanagari style used for writing the denomination 2000. This paper in the second half makes a elaborative assessment of the note with the scanned and photocopied version of the same. Various decisive security features like- Intaglio 
printing, latent image, optically variable ink and optical thread, magic images behind the motif of Mangalyaan are visible in the genuine currency note, and are not appreciated in the scanned and the photocopied note.

When it comes to forgery, the counterfeiting of this highest denomination becomes challenging, because of the presence of various micro security features which makes the note strong and secure. Thus very few probabilities arise to counterfeit the note. Also if the general public is aware of some most common and yet features that remain intact will definitely aid in thwarting forgery of the notes.

\section{Discussion}

This paper has made a small effort in deciphering the security features of the highest Denomination 2000 currency note which has been recently issued by the Government of India. The currency note shares some similar features with the other denomination currencies around the world in respect to the Micro-printing, 3D Security thread, Watermarks, Colour shifting Ink (Optically Variable ink), Raised printing and many more $[6,7,8]$. Counterfeiting the note with all the above mentioned features is impossible. Moreover the denomination can be added with some new weaving pattern or any hidden trait in the blank white portion of the currency note in forgery.

\section{Conclusion}

Due to lack of knowledge it is easy to cheat the common man with fake currency notes. So the present study gives detailed information about the various security features that are embedded in the genuine Indian currency note of the denominational value 2000 which are easy to identify and can be easily identified and observed with the naked eyes. This study also reveals the features that do not appear in the photocopy and scanned currency notes so as to provide a better knowledge and idea about difference in the genuine and fake currency. Every individual must have knowledge about these security features in order to prevent the counterfeiting of the currency notes and to also prevent oneself from being cheated by anyone.

\section{References}

1. First Post (2016) Rs 500 and Rs 1,000 banned: New Rs 2,000 currency note to feature Mangalyaan, raised bleed lines, November 9th India.

2. Financial Express (2016), Rs 2000 currency note by RBI: 15 must-know salient features and how you can identify it, November 9th India.

3. Samudranil (2016) What's Unique About The New Rs 500, Rs 2000 Notes? November 9th, Maps of India.

4. Rubia, Aman B (2016) Security Features In Indian500 Rupee Note. IJESRT 5: 782-85.

5. Mintage World (2016) Security features on Indian Banknotes, May 18th India.

6. U.S. Currency education Program (2013) Currency note of denomination dollar 100.

7. Bank of Japan (2004) Security Features of the New Bank of Japan Notes.

8. The Euro: Our Money. Euro Bank Notes-n Security features.

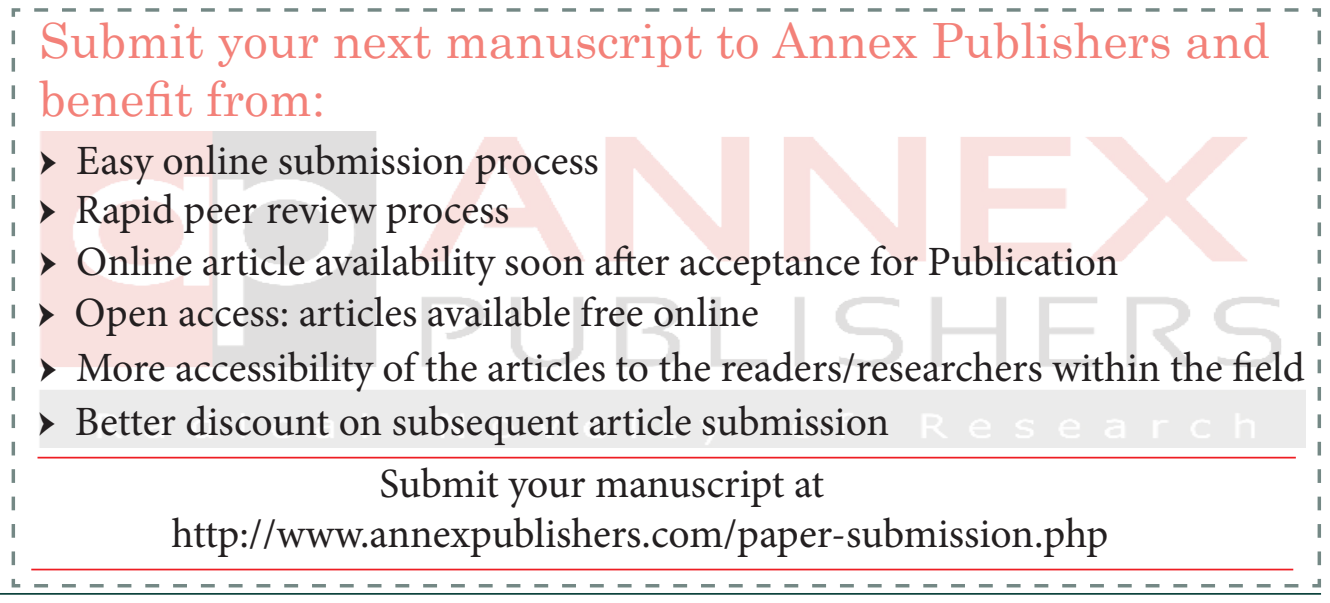

